\title{
O COMPORTAMENTO DE CRIANÇAS EM TRATAMENTO ODONTOLÓGICO: INTERVENÇÃO PSICOFARMACOLÓGICA
}

\author{
Rosana de Fátima Possobon \\ Antonio Bento Alves de Moraes" \\ Gláucia Maria Bovi Ambrozano" \\ Áderson Luiz Costa Junior ${ }^{\circledast 2}$
}

\begin{abstract}
RESUMO. Este trabalho avaliou os efeitos do diazepam sobre os comportamentos de crianças com história de nãocolaboração a tratamento odontológico. Foi administrado placebo ou droga $(0,3 \mathrm{mg} / \mathrm{kg}$ peso) a seis crianças e um total de 54 sessões foram gravadas em vídeo. A análise do registro comportamental dos participantes (choro, movimentos de corpo e/ou cabeça, fuga e esquiva) e das estratégias de manejo (distração, explicação e reforçamento positivo) sistematicamente adotadas não apontou diferença estatística significativa entre sessões placebo e diazepam pelo teste de Wilcoxon pareado ( $p>0,05)$. Sugere-se a necessidade de refinamento metodológico em estudos que combinem estratégias de manejo psicológicas e farmacológicas.
\end{abstract}

Palavras-chave: crianças não-colaboradoras, odontologia comportamental, Diazepam.

\section{CHILDREN'S BEHAVIOR IN DENTISTRY TREATMENT: PSYCHOPHARMACOLOGICAL INTERVENTION}

\begin{abstract}
The present investigation evaluated the effects of diazepam used to manage uncooperative behavior of child dental patients. Six participants received placebo or diazepam $(0,3 \mathrm{mg} / \mathrm{kg}$ weight $)$ before formal dental treatment at total 54 sessions that were all recorded in videotapes. The analysis of recorded child (crying, body and/or head movements, escape and avoidance) and dentist's behavior management procedures (distraction, explanation, positive reinforcement) indicates no differences by using a double-blind Wilcoxon design $(\mathrm{p}>0.05)$. It is suggested the necessity of methodological refinement in studies that combine psychological and pharmacological handling strategies.
\end{abstract}

Key words: uncooperative child; Behavioral dentistry; Diazepam.

\section{INTRODUÇÃO}

$\mathrm{O}$ atendimento odontológico tem sido descrito pela literatura como um contexto potencialmente estressante. Segundo Thom, Sartory e Johren (2000), o medo de dentista constitui uma queixa prevalente em grande parte da população mundial. Nos Estados Unidos da América, por exemplo, 80\% dos adultos relatam algum grau de apreensão quando expostos ao tratamento odontológico. Estatísticas semelhantes foram referidas para populações européias e asiáticas, onde mais de $20 \%$ dos pacientes adultos apresentam alto grau de ansiedade e, pelo menos, 5\% também evitam submeter-se a tratamento odontológico.

Observa-se que este contexto estressante do tratamento odontológico atinge, em especial, a criança, que fica temporariamente exposta a uma situação diferente das experiências cotidianas, a qual envolve equipamentos e instrumentos que, dependendo da rotina odontológica em execução e da habilidade dos profissionais, podem provocar desconforto físico, como a dor, e desconforto psicológico, que inclui elementos de ansiedade e o

* Doutora em Odontologia pela Faculdade de Odontologia de Piracicaba (FOP) - Universidade Estadual de Campinas/Unicamp.

\# Doutor em Ciências pela FOP-Unicamp (Livre-docente em Psicologia pela FOP-Unicamp), Professor Titular da Área de Psicologia Aplicada da Faculdade de Odontologia de Piracicaba/Unicamp.

II Doutora em Ciências pela ESALQ-USP (Livre-docente em Bioestatística pela Unicamp), Professora Associada da Área de Bioestatística da Faculdade de Odontologia de Piracicaba/Unicamp.

æ Doutor em Psicologia pela UnB, Professor Adjunto do Instituto de Psicologia da Universidade de Brasília / UnB. 
medo (Stokes \& Kennedy, 1980; Berge, Veerkamp \& Hoogstraten, 1999). Tal descrição parece justificar a freqüência significativa de mais de $25 \%$ de crianças que apresentam, sistematicamente, comportamentos de não-colaboração com o tratamento odontopediátrico, especialmente padrões de fuga e esquiva, que dificultam ou impedem a adequada atuação do cirurgião-dentista (Possobon, Moraes, Costa Jr. \& Ambrozano, 2003).

Assim, muitos profissionais enfrentam dificuldades para exercer controle sobre comportamentos que facilitem a execução do tratamento (Ayer \& Corah, 1984; Kuhn \& Allen, 1994). Berge e cols. (1999), por exemplo, ressaltam que a utilização freqüente, pelo cirurgião-dentista, de estratégias que envolvam coerção e negação dos sentimentos da criança exposta ao tratamento, não promove a aquisição de comportamentos colaborativos e ainda pode potencializar reações de medo. Por outro lado, Weinstein (1986) já observou que a adoção de estratégias relacionadas ao fornecimento prévio de informação, direcionamento de conduta e compreensão das reações emocionais das crianças facilitava a aquisição e manutenção de comportamentos colaborativos com o tratamento. $\mathrm{O}$ autor destacou ainda que profissionais treinados teriam condições de ajustar o tratamento odontológico ao nível de não-colaboração manifestado pela criança, fornecendo a quantidade de orientação e o suporte necessário para cada indivíduo enfrentar a sequiência de tratamentos programada.

Estatísticas apontam que cada paciente que apresenta ansiedade, quando exposto à situação de consultório, onera o tempo de duração do tratamento odontológico em, pelo menos, $20 \%$ (Thom \& cols., 2000). Em odontopediatria, Ingersoll (1982) já observava que as dificuldades e os riscos envolvidos no atendimento odontológico de crianças nãocolaboradoras ultrapassavam a esfera técnica da odontologia e podiam ser tão evidentes que desencorajariam alguns clínicos de atendê-las.

A atuação profissional do odontopediatra durante $\mathrm{o}$ atendimento de crianças, especialmente daquelas que tendem a não colaborar com o tratamento, requer conhecimento específico para identificar e lidar com variáveis comportamentais, considerando as características de cada criança, as fases de desenvolvimento em que estas se encontram e as circunstâncias de cada rotina odontológica em vigor (Costa Jr. \& Coutinho, 2000).

Neste sentido, a odontologia tem buscado recursos junto à psicologia a fim de definir um conjunto de estratégias para o manejo de comportamentos de pacientes que apresentam dificuldades em enfrentar um tratamento odontológico (Kuhn \& Allen, 1994). Entretanto, algumas crianças mostram-se refratárias ou mais resistentes a estas estratégias, de modo que não foi possível, até o momento, apontar estratégias comportamentais que fossem absolutamente eficientes em controlar o comportamento de crianças expostas a procedimentos médicos e odontológicos invasivos (Costa Jr., 2001).

Nos casos em que o uso exclusivo de estratégias comportamentais não permitiu a plena execução do tratamento odontológico, principalmente nas situações em que o paciente não usufruiu os benefícios esperados do controle comportamental, o emprego de substâncias farmacológicas, tais como calmantes e ansiolíticos, pode constituir uma alternativa para a redução de movimentos motores e de reações indesejáveis do paciente (Coté, 1994), indicando uma diminuição do grau de ansiedade percebido e permitindo a realização do tratamento. Para Moraes e Pessotti (1985), a ansiedade deveria ser compreendida como um componente importante, uma condição estabelecedora para as reações de não-colaboração do paciente. Procedimentos específicos de sedação consciente permitem, ainda, que o paciente mantenha reações apropriadas a estímulos físicos e comandos verbais do cirurgião-dentista (Coté, 1994).

$\mathrm{O}$ emprego de agentes farmacológicos parece ser uma alternativa também nos casos em que não há tempo para o uso de estratégias comportamentais de manejo, como, por exemplo, quando existe urgência na resolução do caso pela presença de sintomatologia dolorosa intensa. Pesquisas com a utilização de sedação leve em pacientes odontopediátricos obtiveram uma redução do tempo operatório de cada sessão, proporcionando um menor grau de agitação, redução da frequiência de choro e aumento dos níveis de sonolência e de cooperação (Badalaty, Houpt, Koenigsberg, Maxwell \& Desjardins, 1990; Coté, 1994; Sams, Cook, Jakson \& Roebuck, 1993).

Alguns trabalhos de pesquisa investigaram os efeitos da combinação de estratégias comportamentais e farmacológicas e demonstraram que esta pode ser uma alternativa viável para a obtenção de controle comportamental sobre pacientes não-colaboradores (Thom \& cols., 2000; Weinstein, 1986).

Dentre os inúmeros agentes farmacológicos que podem ser empregados com a finalidade de aumentar a cooperação potencial de pacientes odontopediátricos, os benzodiazepínicos, em especial o diazepam, têm sido bastante estudados. Eles formam um grupo de drogas com ação ansiolítica, miorrelaxante, sedativa, com efeito amnésico e anticonvulsivante, indicado 
como medicação pré-anestésica no alívio sintomático da ansiedade, agitação e tensão motora devidas a estados psiconeuróticos e distúrbios passageiros induzidos por situação estressante (Fuchs \& Wannmacher, 1992).

Segundo Mandelli, Tognoni e Garattini (1978), o diazepam demonstrou constituir-se em uma modalidade de sedativo particularmente segura e eficaz para pacientes pediátricos, embora apresente a desvantagem da duração de sua ação. Alguns trabalhos posteriores (Flaitz, Nowak \& Hicks, 1989; Simkey, Akyuz, Sener, Göker \& Güvener, 1993; Yanase, Braham, Fukuta \& Kurosu, 1996) também apontam efeitos satisfatórios dos benzodiazepínicos sobre o comportamento das crianças, além de uma grande margem de segurança, o que facilita a aceitação da medicação pelos pais (Lawrence, McTigue, Wilson, Odom, Waggoner \& Fields, 1991; Murphy, Fields \& Machen, 1984). Todavia, outros trabalhos (Fell, Gough, Northan \& Henderson, 1985; Lindsay \& Yates, 1985) não observaram aumento da freqüência de comportamentos cooperativos com o tratamento, talvez pelo uso de dosagens excessivamente reduzidas de diazepam.

Assim sendo, o objetivo deste trabalho foi avaliar a eficácia do uso do diazepam, quando combinado com estratégias psicológicas, no controle do comportamento de crianças não-colaboradoras durante a situação de atendimento odontológico.

\section{METODOLOGIA}

Seis crianças, com idades entre quatro e cinco anos completos, receberam tratamento odontológico durante nove sessões consecutivas. Todas as crianças, atendidas pelo mesmo cirurgião-dentista, apresentavam história anterior de não-colaboração que impedia a realização de tratamentos.

O nível de cooperação da criança foi avaliado com base na escala HBCD, desenvolvida por Allard e Stokes (1980), em uma sessão de atendimento odontológico na qual foram realizados procedimentos clínicos não-invasivos de exame clínico e treinamento de escovação. Foram incluídas na amostra deste estudo as crianças que apresentaram comportamentos de não-colaboração superiores a $25 \%$ do tempo de duração dessa sessão. O HBCD avalia quatro dimensões de comportamentos não-colaborativos: movimentos de cabeça, que atrapalham ou impedem a atuação do cirurgião-dentista; (b) movimentos corporais da criança, que interrompem a rotina odontológica em andamento; (c) freqüência de choro e queixas verbais da criança; e (d) outros comportamentos da criança que interrompem a ação do cirurgião-dentista por períodos de tempo maiores que cinco segundos.

Todo o atendimento odontológico foi realizado nas dependências do Laboratório de Psicologia Aplicada (LPA), uma unidade do Centro de Pesquisa e Atendimento Odontológico para Pacientes Especiais (Cepae) da Faculdade de Odontologia de Piracicaba Unicamp. O projeto foi aprovado pelo Comitê de Ética em Pesquisa dessa Faculdade.

Os participantes foram divididos aleatoriamente em dois grupos, designados Grupo A e Grupo B. Na primeira sessão não foi administrado nenhum medicamento. A partir da segunda sessão, cada participante recebeu, de maneira duplo-cego, o diazepam ou placebo. A dose do diazepam foi de $0,3 \mathrm{mg} / \mathrm{kg}$ de peso, administrada por via oral à criança uma hora antes do início da sessão. O placebo foi administrado em igual volume e via do diazepam, também uma hora antes do início da sessão.

Os participantes de cada grupo receberam diazepam ou placebo na seqüência apresentada no Quadro 1.

Quadro 1. Esquema representativo do delineamento experimental .

\begin{tabular}{|c|c|c|}
\hline Sessão & Grupo a & Grupo b \\
\hline $\mathbf{1}^{\mathbf{a}}$ & Sem droga & Sem droga \\
\hline $\mathbf{2}^{\mathbf{a}}$ & Placebo & Diazepam \\
\hline $\mathbf{3}^{\mathbf{a}}$ & Placebo & Diazepam \\
\hline $\mathbf{4}^{\mathbf{a}}$ & Diazepam & Placebo \\
\hline $\mathbf{5}^{\mathbf{a}}$ & Diazepam & Placebo \\
\hline $\mathbf{6}^{\mathbf{a}}$ & Placebo & Diazepam \\
\hline $\mathbf{7}^{\mathbf{a}}$ & Placebo & Diazepam \\
\hline $\mathbf{8}^{\mathbf{a}}$ & Diazepam & Placebo \\
\hline $\mathbf{9}^{\mathbf{a}}$ & Diazepam & Placebo \\
\hline
\end{tabular}

Em todas as sessões de atendimento, o cirurgiãodentista adotou, sistematicamente, as seguintes estratégias psicológicas de manejo de comportamentos:

1) Distração: consistiu em introduzir, no ambiente odontológico, novos estímulos que apresentam o potencial de desviar a atenção do paciente, de elementos aversivos típicos do consultório, em direção a situações imaginárias e/ou incompatíveis com a execução do tratamento e com a dor ou tensão nervosa, conforme descrito por Stark, Allen, Hurst, Nash e Rigney (1989). Neste trabalho, o cirurgião-dentista conversava ou cantava para a criança, na tentativa de desviar sua atenção de cada 
uma das rotinas odontológicas executadas na sessão.

2) Explicação: consistiu na adoção da estratégia de "conte-mostre-faça", conforme descrita por Addelston (1959), além de solicitações verbais de colaboração sempre que um comportamento nãocolaborativo era apresentado pela criança. A estratégia clássica do "conte-mostre-faça" envolve um procedimento rotineiro de três etapas: (a) explicar previamente um procedimento ou descrever um equipamento que será utilizado; (b) apresentar o equipamento ou instrumento à criança; e (c) executar o procedimento após consentimento da criança.

3) Reforçamento positivo: consistiu na apresentação de estímulos reforçadores verbais, tais como elogios e incentivos para manter-se colaborativa, imediatamente após a apresentação de comportamentos compatíveis com a execução do tratamento, conforme o descrito por Moraes e Pessotti (1985).

Todas as 54 sessões, isto é, nove sessões para cada um dos seis participantes, foram gravadas em vídeo, com sons de "bip" a cada 15 segundos, que marcavam os momentos em que, posteriormente, foram efetuados os registros dos comportamentos observados, conforme procedimento descrito por Allard e Stokes (1980) e Stokes e Kennedy (1980). As categorias de comportamentos da criança, observadas e registradas a partir dos dados obtidos em vídeo, foram as seguintes:

1) Movimentos de corpo e/ou cabeça: manifestados durante a execução de um procedimento odontológico que atrapalhasse a atuação do dentista, porém, sem interromper tal procedimento;

2) choro: registrado somente quando ocorria isoladamente; se a criança estivesse chorando e, ao mesmo tempo, apresentando outros comportamentos, o choro não era registrado;

3) fuga: interrupção de um procedimento clínico, por meio de movimentos bruscos de corpo e/ou cabeça, na presença, ou não, de choro ou reclamações;

4) esquiva: seqüência de comportamentos que não permitiam o início da execução de um determinado procedimento; por exemplo, quando a criança não seguia instruções, recusando-se a se deitar na cadeira ou a abrir a boca.

As sessões foram assistidas por dois pesquisadores, que efetuavam registros, individuais e independentes, das estratégias adotadas pelo cirurgiãodentista e dos comportamentos apresentados pelas crianças, utilizando uma folha de registro especialmente desenvolvida para este estudo. Os registros foram comparados a fim de se avaliar o nível de concordância entre observadores, obtendo-se uma média de $93 \%$ de fidedignidade.

Os dados obtidos de todas as sessões com diazepam e de todas as sessões com placebo, de ambos os grupos, foram agrupados e efetuou-se o teste estatístico de Wilcoxom pareado.

\section{RESULTADOS}

Para análise dos resultados, as respostas comportamentais das crianças foram agrupadas funcionalmente em "fuga/esquiva" (momentos em que a criança não permitia a atuação do cirurgião-dentista) e "choro/movimentos de corpo" (comportamentos que atrapalham, mas não impedem a realização do tratamento).

A Tabela 1 apresenta os resultados obtidos em relação aos comportamentos das crianças e demonstra não ter havido diferença estatisticamente significativa entre as sessões nas quais as crianças receberam placebo e aquelas em que receberam o diazepam, pelo teste de Wilcoxon pareado $(\mathrm{p}>0,05)$ para todas as variáveis.

Tabela 1. Freqüência média dos comportamentos emitidos pelos participantes nas sessões com placebo e nas sessões com diazepam. As médias seguidas de letras iguais, na horizontal, não diferem entre si pelo teste de Wilcoxon pareado $(\mathrm{p}>0,05)$.

\begin{tabular}{lll}
\hline \multirow{2}{*}{ Comportamentos } & \multicolumn{2}{c}{ Medicação } \\
\cline { 2 - 3 } & \multicolumn{1}{c}{ Placebo } & Diazepam \\
\hline Choro e movimentos & $17,05 \mathrm{a}$ & $16,95 \mathrm{a}$ \\
\hline Fuga e esquiva & $26,08 \mathrm{~b}$ & $26,87 \mathrm{~b}$ \\
\hline
\end{tabular}

Analisando-se individualmente o desempenho de cada criança, não se observou, com exceção de um participante que apresentou acentuada sonolência, nenhuma diferença significativa no repertório de comportamentos que pudesse ser atribuída ao medicamento ansiolítico. Notou-se, apenas, a ocorrência de pequenos episódios de relaxamento muscular, especialmente no final das sessões em que o diazepam foi administrado.

Os comportamentos de movimentos de corpo e/ou cabeça e choro ocorreram, com maior freqüência, durante a execução de rotinas odontológicas descritas como potencialmente mais aversivas, isto é, anestesia injetável e preparo cavitário. Considerando-se as mesmas rotinas odontológicas, não se observaram 
diferenças significativas de comportamentos entre sessões com administração de diazepam e placebo.

A Tabela 2 apresenta os resultados obtidos em relação às estratégias adotadas pelo cirurgião-dentista e demonstra não ter havido diferença significativa entre as sessões nas quais as crianças receberam placebo e aquelas em que receberam o diazepam, pelo teste de Wilcoxon pareado $(\mathrm{p}>0,05)$ para todas as variáveis. $\mathrm{O}$ cirurgião-dentista adotou, em proporções semelhantes, as mesmas estratégias psicológicas, independentemente da condição da sessão - ansiolítico ou placebo.

Tabela 2. Frequiência média das estratégias psicológicas adotadas pelo cirurgião-dentista nas sessões com placebo e nas sessões com diazepam. As médias seguidas de letras iguais, na horizontal, não diferem entre si pelo teste de Wilcoxon pareado $(\mathrm{p}>0,05)$.

\begin{tabular}{lll}
\hline \multirow{2}{*}{ Comportamentos } & \multicolumn{2}{c}{ Medicação } \\
& Placebo & Diazepam \\
\hline Explicação & $56,03 \mathrm{c}$ & $54,14 \mathrm{c}$ \\
\hline Distração e elogio & $27,51 \mathrm{~d}$ & $28,08 \mathrm{~d}$ \\
\hline
\end{tabular}

Observou-se que verbalizações elogiosas do cirurgião-dentista, a comportamentos colaborativos apresentados pelas crianças, independentemente da rotina odontológica em andamento, forampercebidas pelas crianças como fator favorável ao estabelecimento e manutenção das relações profissional-cliente. Mesmo sob a execução de rotina geralmente percebida como aversiva, como a anestesia injetável, um elogio do cirurgião-dentista era seguido por um movimento de olhar da criança que parecia aliviar, temporariamente, seu sofrimento percebido, reduzindo-se a intensidade da agitação motora ou o volume de choro em momentos imediatamente posteriores.

\section{DISCUSSÃO}

Como os resultados mostraram não ter havido diferenças entre as sessões com placebo e com diazepam, em relação aos comportamentos de fuga e esquiva, não se confirmou a expectativa de que, com o emprego do diazepam, as crianças tivessem uma maior freqüência de respostas de colaboração, isto é, manifestassem menos freqüentemente comportamentos de fuga e esquiva. Em função de os episódios de sonolência terem sido observados em apenas um participante, sugere-se que a dose empregada tenha sido insuficiente para alterar $o$ repertório de comportamentos não-colaborativos das crianças.

$\mathrm{Na}$ dose utilizada, a medicação parece ter apresentado algum efeito redutor apenas sobre os comportamentos de um dos participantes do Grupo A, que chorava e movimentava-se, mas não apresentava respostas de fuga e esquiva, o que permitiu a realização do tratamento. Vale ressaltar que este participante não foi o mesmo que se apresentou sonolento durante as sessões com diazepam. Embora não-colaborador, este participante apresentava um padrão de comportamento diferente daquele dos demais, emitindo maior freqüência de comportamentos que não interrompiam a execução de procedimentos do cirurgião-dentista, isto é, choro e movimentos de corpo.

Outros estudos também mostraram a ineficácia do diazepam quando empregado em doses reduzidas. Fell, Gough, Northan e Henderson (1985), por exemplo, analisaram 101 crianças, utilizando doses de 0,25 e $0,5 \mathrm{mg} / \mathrm{kg}$ de diazepam, em que não se revelaram modificações significativas no padrão de comportamentos das crianças. Lindsay e Yates (1985) também não verificaram diferenças entre as sessões com placebo e com diazepam, utilizando doses de $0,2 \mathrm{mg} / \mathrm{kg}$ de peso.

Por outro lado, há diversos estudos que relataram a influência do diazepam sobre o aumento da colaboração de crianças expostas a tratamento odontológico. Yanase, Braham, Fukuta, e Kurosu (1996) relataram $60 \%$ de aumento na frequiência de ocorrência de comportamentos colaborativos, utilizando-se diazepam na dose de $0,3 \mathrm{mg} / \mathrm{kg}$, em comparação com as sessões em que as crianças receberam placebo. No entanto, apesar do aumento do grau de cooperação, os pacientes ainda apresentavam alguns comportamentos incompatíveis com uma adequada atuação do dentista, incluindo movimentos físicos e protestos verbais. Flaitz, Nowak e Hicks (1989) descreveram uma redução significativa na freqüência de comportamentos de não-colaboração em doze crianças expostas a tratamento odontológico, quando foram comparadas sessões em que se administrou placebo e se administrou $0,6 \mathrm{mg} / \mathrm{kg}$ de diazepam.

Tais dados sugerem uma necessidade de ajuste da dose do medicamento ansiolítico para cada paciente, de maneira individualizada, iniciando por uma dose mínima que poderia ser $0, \mathrm{mg} / \mathrm{kg}$ de peso, uma vez que esta parece ter sido eficaz para um dos participantes deste estudo. Para o ajuste da dose, devem ser consideradas algumas características do paciente: (a) a história de experiências do indivíduo 
com tratamento odontológico; (b) o padrão comportamental de não-colaboração - relatado pelos pais e observado pelo profissional; e (c) os indicadores comportamentais e cognitivos de ansiedade antecipatória, avaliados em momentos anteriores ao início das sessões de atendimento.

Para ter sido considerada eficaz, a medicação não precisaria ter provocado sedação que levasse a criança a dormir durante toda a sessão. O objetivo do emprego do ansiolítico é tranqüilizar a criança, deixando-a mais receptiva às estratégias de manejo comportamental empregadas, possibilitando a realização plena do tratamento no menor tempo possível. Thom e cols. (2000) sugeriram que o ansiolítico pode ser empregado como um coadjuvante das estratégias psicológicas, com o objetivo de facilitar a exposição dos participantes às contingências naturais do tratamento e diminuir as propriedades aversivas dos estímulos presentes na situação de atendimento odontológico.

Nenhum efeito colateral foi registrado durante e após o encerramento das sessões, quando as crianças foram continuamente observadas, por suas respectivas mães, na sala de espera, por um período aproximado de meia hora, confirmando a margem de segurança da administração do diazepam (Coté, 1994). Ressalte-se que as mães também foram orientadas a observar os comportamentos de seus filhos em casa, informando, na sessão subseqüente, qualquer condição considerada diferente do usual. Tal como na primeira meia hora pós-sessão, não houve qualquer episódio comportamental considerado estranho por parte das mães.

\section{CONSIDERAÇÕES FINAIS}

O diazepam, utilizado na dose de $0,3 \mathrm{mg} / \mathrm{kg}$, administrado uma hora antes do início das sessões, mostrou-se ineficaz para um efetivo controle de comportamentos de não-colaboração com o tratamento, não se observando diferenças significativas no grau de colaboração dos participantes, quando comparadas as sessões com medicação e com placebo. Este resultado não invalida, contudo, a utilização de ansiolíticos como potenciais redutores de comportamentos não-colaborativos com tratamento odontológico. É necessário chamar a atenção para a necessidade de se avaliarem possíveis efeitos específicos da administração de diferentes doses de diazepam, bem como a influência da história de experiências da criança com a situação odontológica e o grau de não-colaboração manifestado em condição anterior a qualquer manipulação ambiental e/ou farmacológica.

As estratégias psicológicas de manejo do comportamento, como a distração e a explicação, devem ser utilizadas em todas as sessões de atendimento odontopediátrico. Sugere-se que a adoção destas estratégias amenize os efeitos aversivos da situação de tratamento, especialmente de rotinas odontológicas invasivas, fazendo com que a criança se sinta amparada e perceba maior controle da situação. A atenção incondicional ao paciente - exposto aos mais diversos tipos de sensações, algumas desagradáveis - parece contribuir para o aumento da confiança no profissional de saúde, e conseqüentemente, para a melhoria da relação profissional-paciente, fator essencial à obtenção de controle sobre os comportamentos da criança.

Outros estudos, desenvolvidos na interface odontologia-psicologia, poderiam considerar delineamentos que combinassem estratégias comportamentais e farmacológicas, utilizando-se formas sistemáticas de medidas pontuais dos comportamentos, permitindo o registro minucioso dos efeitos da adoção de estratégias comportamentais e cognitivas em rotinas odontológicas específicas.

\section{REFERÊNCIAS}

Addelston, H.K. (1959). Child patient training. Fortnightly Review of the Chicago Dental Society, 38, 7-11.

Allard, G. \& Stokes, T. F. (1980). Continuous observation: a detailed record of children's behavior during dental treatment. Journal of Dentistry for Children, 47, 246-250.

Ayer, W.A. \& Corah, N.L. (1984). Behavioral factors influencing dental treatment. Em L.K. Cohen \& P.S. Bryant (Orgs.), Social sciences and dentistry: a critical bibliography - V.II. (pp. 267-322). Berlim: Quintessence

Badalaty, M.M., Houpt, M.I., Koenigsberg, S.R., Maxwell, K.C. \& Desjardins, P.J. (1990). A comparison of chloral hidrate and diazepam sedation in young children. Pediatric Dentistry, 12, 33-37.

Berge, M.T., Veerkamp, J. \& Hoogstraten, J. (1999). Dentist's behavior in response to child dental fear. Journal of Dentistry for Children, 63, 36-40.

Costa Jr., A. L. (2001). Análise de comportamentos de crianças expostas à punção venosa para quimioterapia. Tese de Doutorado, não-publicada, Programa de Pós-Graduação em Psicologia do Escolar e do Desenvolvimento, Instituto de Psicologia. Universidade de Brasília.

Costa Jr., A.L. \& Coutinho, S.M.G. (2000). Você tem medo de dentista? A psicologia pode ajudar-lhe. Leia: Informação $e$ Crítica, 2 (9), 20-24.

Coté, C.J. (1994). Sedation for the pediatric patients: a review. The Pediatric Clinics of North America, 41, 31-58. 
Fell, D., Gough, M.B., Northan, A.A. \& Henderson, C.U. (1985). Diazepam premedication in children: plasma levels and clinical effects. Anaesthesia, 40, 12-17.

Flaitz, C., Nowak, A. \& Hicks, M.J. (1989). Evaluation of the anterograde amnesic effect of rectally administered diazepam in the sedated dental patient. Journal of Dentistry for Children, 53, 17-20.

Fuchs, F. D. \& Wannmacher, L. (1992). Farmacologia clínica: fundamentos para terapêtica racional. Rio de Janeiro: Guanabara Koogan.

Ingersoll, B.D. (1982). Behavioral aspects in dentistry. (pp. 107-133). New York: Appleton-Century - Crofts.

Kuhn, B.R. \& Allen, K.D. (1994). Expanding child behavior management technology in pediatric dentistry: a behavioral science perspective. Pediatric Dentistry, 16, 13-17.

Lawrence, S.M., McTigue, D. J., Wilson, S., Odom, J.G., Waggoner, W.F. \& Fields, H.W. (1991). Parental attitudes toward behavior management techniques used in pediatric dentistry. Pediatric Dentistry, 13, 151-155.

Lindsay, S.J.E. \& Yates, J.A. (1985). The effectiveness of oral diazepam in anxious child dental patients. British Dentistry Journal, 159 (5), 149-153.

Mandelli, M., Tognoni, G. \& Garattini, S. (1978). Clinical pharmacokinetics of diazepam. Clinical Pharmacokinetics, 3, 72-91.

Moraes, A.B.A. \& Pessotti, I. (1985). Psicologia aplicada à Odontologia. São Paulo: Sarvier.

Murphy, M.G., Fields, H.W. \& Machen, J.B. (1984). Parental acceptance of pediatric dentistry behavior management techniques. Pediatric Dentistry, 6, 193-198.

Possobon, R.F., Moraes, A.B.A., Costa Jr., A.L. \& Ambrozano, G.M.B. (2003). O comportamento de crianças durante atendimento odontológico. Psicologia: Teoria e Pesquisa, $19,59-64$.
Sams, D.R., Cook, E.W., Jakson, J.G. \& Roebuck, B.L. (1993). Behavioral assessment of two drug combinations for oral sedation. Pediatric Dentistry, 15, 186-190.

Simkey, S., Akyuz, S., Sener, G., Göker, K. \& Güvener, Ö. (1993). Classification and premedication of uncooperative children. The Journal of Nihon University School of Dentistry, 5, 28-35.

Stark, L.J., Allen, K.D., Hurst, M., Nash, D.A. \& Rigney, B. (1989). Distraction: its utilization and efficacy with children undergoing dental treatment. Journal of Applied Behavior Analysis, 22, 297-307.

Stokes, T.F. \& Kennedy, S.H. (1980). Reducing child uncooperative behavior during dental treatment through modeling and reinforcement. Journal of Applied Behavior Analysis, 13, 41-49.

Thom, A., Sartory, G. \& Johren, P. (2000). Comparison between one-session psychological treatment and benzodiazepine in dental phobia. Journal of Consulting and Clinical Psychology, 68, 378-387.

Weinstein, P. (1986). Integrating behavioral methodologies into Dental Pharmacological Research. Anesthesia Progress, 33, 55-59.

Yanase, H., Braham, O.F., Fukuta, O. \& Kurosu, K. (1996). A study of the sedative effect of home-administered oral diazepam for the dental treatment of children. International Journal of Paediatric Dentistry, 6, 13-17.

Recebido em 15/08/2003 Aceito em 05/03/2004

Endereço para correspondência: Rosana de Fátima Possobon, Av. Limeira, 901, Areião, Caixa Postal 52, CEP: 13414-903, Piracicaba-SP. E-mail: possobon@fop.unicamp.br. 